\title{
AN INVESTIGATION OF FATIGUE PROPERTIES OF PRE-QUENCHED- TEMPERED S45C STEEL WITH PLASMA NITRIDING TREATMENT
}

\author{
Bo $\mathrm{Wu}$
}

State key laboratory for mechanical behavior of materials, Xian Jiaotong University, Xian 710049, China Department of Mechanical Engineering, The University of Tokushima, 2-1 Minami-josanjima-cho, Tokushima, Japan

walkman.wb@gmail.com

Jianxun Zhang

State key laboratory for mechanical behavior of materials, Xian Jiaotong University, Xian 710049, China jxzhang@mail.xjtu.edu.cn

Ri-ichi Murakami

Department of Mechanical Engineering, The University of Tokushima, 2-1 Minami-josanjima-cho, Tokushima, Japan

murakami@me.tokushima-u.ac.jp

\begin{abstract}
Quenched and tempered (QT) S45C steel was processed by plasma nitriding (a mixture of $40 \% \mathrm{~N}_{2}$ $60 \% \mathrm{H}_{2}, 400 \mathrm{~Pa}, 773 \mathrm{~K}$ ) with two different durations: $8 \mathrm{~h}$ and $48 \mathrm{~h}$. The microhardness, surface residual stress and nitriding layer compound were separately detected and the effect was discussed with the fatigue test result. The rotating bending fatigue properties was test with QT, QT nitiding 8 $\mathrm{h}$, QT nitriding $48 \mathrm{~h}$ specimens. Fish-eye type crack formation occurred and the two crack initiation modes, fish-eye crack initiation and surface crack initiation, were observed by the scanning electron microscope and discussed. The result showed that the fatigue strength of QT specimens were improved by $54 \%$ at least compared with the untreated specimens. But the QT and un-QT have the mainly same fatigue strength in the same plasma nitriding condition, especially no-improvement with the $48 \mathrm{~h}$ plasma nitriding specimens.
\end{abstract}

Keywords: Fatigue; Residual stress; Plasma nitriding; Fish-eye.

\section{Introduction}

To improve the fatigue strength, surface hardness, tribological and anticorrosion properties of metal, nitriding as one of thermo-chemical diffusion surface process technique, has been used on variety alloys. After nitriding a thin compound layer, a diffusion zone beneath the compound layer will be produced by the reaction of nitrogen with metal and diffusion into the bulk at high temperature. The compound layer which is difficult to be etched enhances the properties of friction wear and anticorrosion, but the resistance of fatigue is strengthened by the hard surface and the diffuse zone for the crack 
initiation of subsurface ${ }^{1}$. The fatigue strength depends on not only the surface hardness but also the depth of diffusion zone and these two parameters are important for improving fatigue properties.

It is known that kinds of parameters, the time duration ${ }^{2}$, treatment temperature ${ }^{3}$, the specific gas composition ${ }^{4}$, the alloying elements and the initial microstructure of the materials all have a great effect on the surface microhardness and the depth of diffusion zone. The process of quenching and tempering (QT) is a widely used treatment to improve the properties of material and get a fine grain size to improve the strength of materials. Before nitriding treatment, all specimens were processed by QT.

In this paper, the main objective is to make quenched and tempered (QT) S45C be nitrided and to study the influence of QT treatment method to plasma nitriding of S45C, especially the microhardness of the surface zone and the depth of diffusion zone to the fatigue strength, with different treatment time durations. The surface layer was detected by XRD to study the compound growing during the nitriding process. SEM and EDX were employed to investigate the fractures and the inclusions inducing fish-eye crack initiation mode in the fatigue test.

\section{Sample preparation and experimental procedures}

The material studied in this paper was one medium carbon steel: S45C of chemical composition (wt \%): $0.45 \% \mathrm{C} ; 0.15 \% \sim 0.35 \% \mathrm{Si} ; 0.6 \sim 0.9 \% \mathrm{Mn} ; \leq 0.03 \% \mathrm{P} ; \leq 0.035 \% \mathrm{~S} ; \leq$ $0.3 \% \mathrm{Cu} ; \leq 0.2 \% \mathrm{Ni} ; \leq 0.2 \% \mathrm{Cr} ;$ balance $\mathrm{Fe}$. Before rotating-bending fatigue test, machining, polishing, quenching-tempering, plasma nitriding were carried out on the specimens to get the standard shape (Fig.1) and a global improved properties.

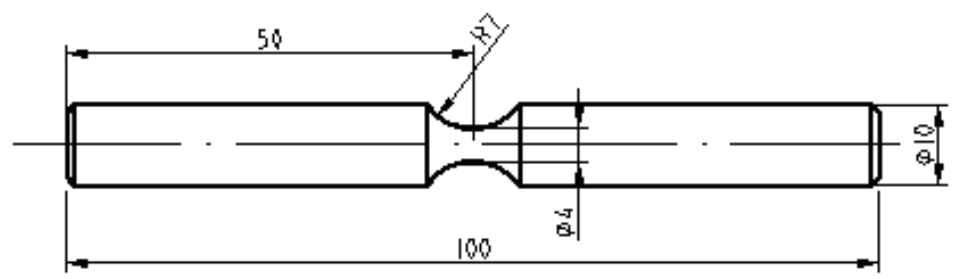

Fig.1.Spesimens of rotating bending fatigue test.

After the specimens polished with sandpapers from grade 120 to grade 2000 and washed in acetone and alcoholic using ultrasonic cleaner, a staged heat mode $\left(400{ }^{\circ} \mathrm{C}-1\right.$ min, $700{ }^{\circ} \mathrm{C}-30 \mathrm{~min}, 840{ }^{\circ} \mathrm{C}-150 \mathrm{~min}$ ) was employed to avoid a heat deformation and austenitize the specimens. Then the specimens were oil-quenched and tempered at $500{ }^{\circ} \mathrm{C}$ for $270 \mathrm{~min}$ to a hardness of $31 \mathrm{HRC}$. The grease and dirt were removed from the specimens' surface by polishing and washing, and then the plasma nitriding process was performed in a mixture gas $\left(40 \% \mathrm{~N}_{2}-60 \% \mathrm{H}_{2}\right)$ at $400 \mathrm{~Pa}$. The specimens were held at 500 ${ }^{\circ} \mathrm{C}$ for two kinds of process time: $8 \mathrm{~h}$ and $48 \mathrm{~h}$. So fatigue property of three kinds specimens, quenched and tempered (QT), QT and plasma nitriding $8 \mathrm{~h}$ (QTPN8), QT and plasma nitriding $48 \mathrm{~h}$ (QTPN48) were test on a rotating bending fatigue test machine with 
dual spindles, which can test four specimens at the same time. The machine runs with a frequency about $52 \mathrm{~Hz}$.

To investigate the micro-character, the specimens was cut from the cross-section direction and polished by sandpaper from grade 150 to grade 1500 , then a polishing cloth with a liquid suspension of alumina on an auto-polish machine. The microhardness measurements of the cross-section specimens were carried out on a Vicker hardness test machine (MMT-7, Matsuzawa) with a load of $100 \mathrm{~g}$ and duration of $10 \mathrm{~s}$. The metallography of the cross-section etched at room temperature by $5 \%$ nital was observed by an optical microscopy to get information of nitriding phase.

The surface residual stress is one important factor impacting fatigue strength, so it was measured by Rigaku Rint-2000 X-Ray diffraction with $\mathrm{CrK}_{\alpha}$ radiation. The method of processing data used the classical $\sin 2 \psi$ through Rigaku PSPC. At the same time, the phases of the nitride layer were also detected by the same machine.

The SEM (Scanning Electron Microscopy S-4700, Hitachi) was employed to observe the fracture surface to distinguish the crack initiation modes and the EDX of the same machine was used to detect the chemical composition of impurity which was the origin of fish-eye crack initiation mode.

\section{Results and discussion}

\subsection{Microstructure features}
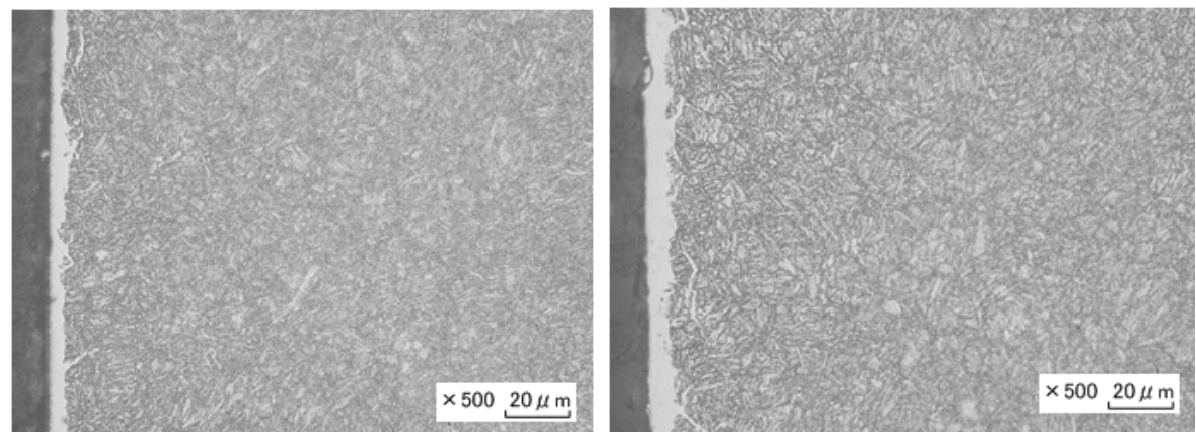

Fig.2. Microstructures of the section-cross of specimens with plasma nitriding $8 \mathrm{~h}$ (left) and $48 \mathrm{~h}$ (right).

Fig. 2 shows the section-cross microstructure of nitriding specimens at two different time durations, $8 \mathrm{~h}$ and $48 \mathrm{~h}$. From the optical microstructures, it can be seen that the white layer is thicker with the nitrding time increasing and beneath the layer the microstructure is a needle-like grain structure for the treatment of quenching and tempering before nitriding. After measuring the thickness of white layer, we can get that the thickness of QTN8 is from $2.83 \mu \mathrm{m}$ to $6.258 \mu \mathrm{m}$ and QTN48 is from $6.75 \mu \mathrm{m}$ to $9.425 \mu \mathrm{m}$. The nonuniform thickness of the white layer depends on the nucleation of new $\mathrm{Fe}_{4} \mathrm{~N}$ precipitates and the branching from the initial precipitates ${ }^{5}$. The average thickness of compound layer increases as nitriding time grows. It is easy to understand that with 
longer process time, more nitrogen has a chemical reaction with iron and the nitrides grow in the high temperature ${ }^{6,7}$.

To study the nitrides of the compound layers, XRD was used to detect the white layer and find that it is mainly consisted by $\gamma^{\prime}$ Fe4N from the result of XRD patterns, Fig. 3 and the result agreed with the previous research ${ }^{7}$.

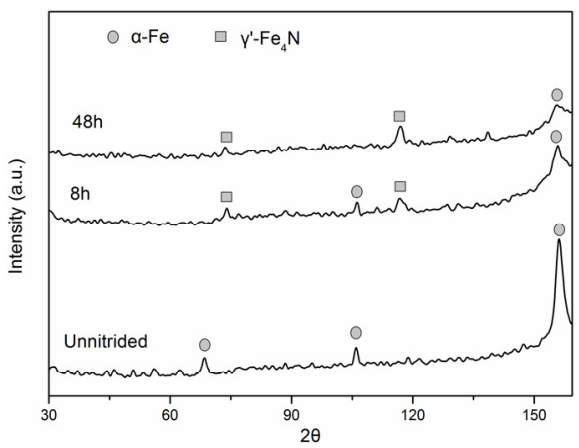

Fig.3. X-ray diffraction patterns of specimens surface with different time durations.

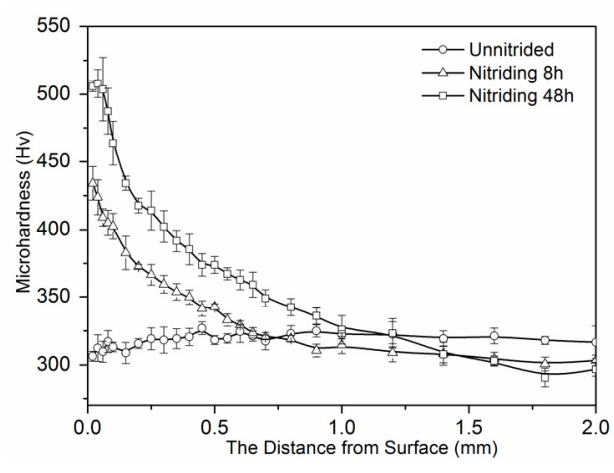

Fig.4. Microhardness of the section-cross of plasma nitriding and unnitrided specimens.

\subsection{Variation of microhardness}

The microhardness of the cross-section was detected from the point $20 \mu \mathrm{m}$ from the treated surface, as the compound layer is too thin to be measured. So the result mainly is the microhardness of diffusion zone. From the Fig.4, it can be seen that the core hardness is smaller than the only QT specimens. This is because that the nitriding process acts as a tempering process in the core of specimens where the nitrogen is difficult to arrive for the nitrides precipitation and compound layer having a resistance of the diffusion of nitrogen.

The depth of diffusion zone was defined as the distance from the surface to the depth where the microhardness is $10 \%$ above the core hardness. So the depth is $0.5 \mathrm{~mm}$ for QTN8 and $0.9 \mathrm{~mm}$ for QTN48. The result also shows that with the nitriding time increasing, the diffusion layer grows. Comparing with the previous work of our research ${ }^{7}$ in which the un-QT specimens were nitrided, the hardness and depth of diffusion zone have a certain increase. The pre-treatment of QT which induces a fine grain can conduce to the nitrogen diffusion in the subsurface of specimens for fine grains containing more grain boundaries providing the main channels for nitrogen diffuse into the core of material.

\subsection{Residual stress distribution}

From Table.1, we can find on the surface of nitrided specimens, compressive residual stress was induced after nitriding. The un-nitrided specimens also have a compressive residual stress because of the QT pre-treatment. During nitriding the residual stress induced by QT was removed at the initial stage and the nitride case tends to expand for nitrides growing, so the compressive residual stress grows. For the un-nitrided core which 
counteracts with the surface residual stress, there will be a tensile stress. As a result normally a compressive residual stress develops near the surface while a tensile residual stress evolves in the core. The residual stress is mainly contributed by the nitriding metallic along the grain boundaries ${ }^{8}$. The residual stress is smaller than the materials containing $\mathrm{Cr}$. The main reason is that $\mathrm{Cr}$ is one chemical element which easily reacts with $\mathrm{N}$ element and change into $\mathrm{CrN}$. For $\mathrm{S} 45 \mathrm{C}$, there is little $\mathrm{Cr}$, so little $\mathrm{CrN}$ is produced and small residual stress is induced.

Table.1. Residual stress on the surface of specimens.

\begin{tabular}{cccc}
\hline Processing method & Un-nitrided & Nitriding $8 \mathrm{~h}$ & Nitriding 48h \\
\hline Residual stress $(\mathrm{MPa})$ & -217.4 & -167.7 & -161.2 \\
\hline
\end{tabular}

\subsection{Enhanced fatigue behaviors}

The fatigue test was carried on rotating bending fatigue machine and the S-N curves were obtained and shown in Fig.5. After nitriding process, the fatigue limit of nitriding $8 \mathrm{~h}$ specimens was $703 \mathrm{MPa}$ and the nitriding $48 \mathrm{~h}$ was $903 \mathrm{MPa}$. From the result of fatigue test, the increasing strength of fatigue property is $100 \%$. The crack initiation can be broken down into two modes, crack surface initiation and fish-eye initiation (marked with vertical bars), Fig.6. The fish-eye crack remains the one of the main crack initiation mode in this test. Through EDX, the element of the inclusions were detected and found that it mainly is aluminum oxide and calcium oxide. The fatigue strength limitation closely connects with the distribution of microhardness, also the depth of diffusion zone. But for the nitriding $48 \mathrm{~h}$ specimens, the same micohardness distribution in the risk region of fish-eye initiation and fatigue strength are performed by QT and Annealed specimens. The nitriding $8 \mathrm{~h}$ specimens having a stronger limitation maybe the result of a deeper diffusion zone induced by the less grain size which was obtained by pre-QT processing.

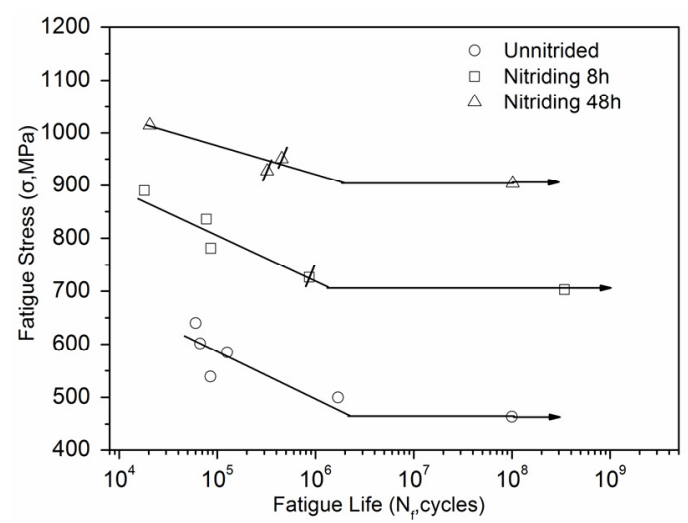

Fig.5. S-N curve of un-nitrided and nitrided specimens. (Fish-eye initiation marked with vertical bars) 

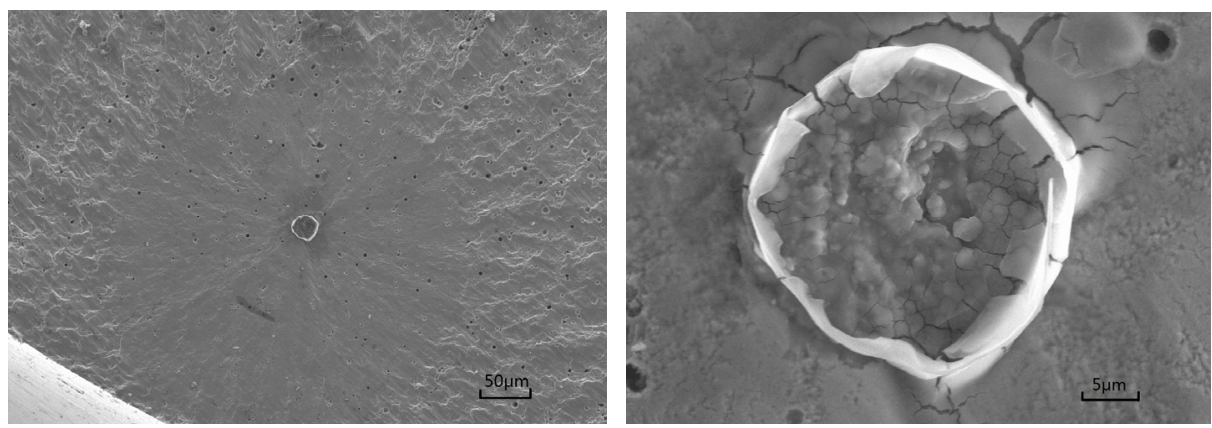

Fig.6. Fish-eye crack initiation and inclusion.

\section{Conclusions}

(1) A compound layer consisted of $\gamma^{\prime}$ Fe4N and a diffusion zone were produced after nitriding process.

(2) The pre-QT improves the fatigue properties of unnitriding and nitriding $8 \mathrm{~h}$ specimens, but for nitriding $48 \mathrm{~h}$ even no improvement.

(3) The nitriding time also is an important factor for a better hard surface, diffusion zone and fatigue properties.

(4) The fish-eye crack initiation was one of the main crack initiation modes when the load stress was close to the fatigue limit stress.

\section{Acknowledgments}

The authors would like to thank Atsushi Onizawa of Neturen Company for the assistant of plasma nitriding and microhardness test.

\section{References}

1. Sun, Y. \& Bell, T. Plasma surface engineering of low alloy steel. Mater. Sci. Eng A 140, 419434 (1991).

2. Genel, K., Demirkol, M. \& Cap, M. Effect of ion nitriding on fatigue behaviour of AISI 4140 steel. Mater. Sci. Eng A 279, 207-216 (2000).

3. Keddam, M. Characterization of the nitrided layers of XC38 carbon steel obtained by R.F. plasma nitriding. A. Surf. Science 254, 2276-2280 (2008).

4. Berg, M. et al. On plasma nitriding of steels. Surf. Coat. Technol. 124, 25-31 (2000).

5. Salas, O., Figueroa, U., Bernal, J. L. \& Oseguera, J. Nitride nucleation and growth during plasma and post-discharge nitriding. Surf. Coat.s Technol. 163-164, 339-346 (2003).

6. Tong, W. P., Liu, C.Z., Tao, N.R., Wang, Z.B., Zuo, L., He, J.C. Gaseous nitriding of iron with a nanostructured surface layer. Scripta Materialia 57, 533-536 (2007).

7. Cao, X. J., Yang, F. \& Murakami, R. Fatigue properties of a S45C steel with plasma nitriding. Auckland (New Zealand), International Conference on Structural Integrity and Failure 7 (SIF 2010).

8. Wiggen, P. C. V., Rozendaal, H. C. F. \& Mittemeijer, E. J. The nitriding behaviour of ironchromium-carbon alloys. J. Mater. Science 20, 4561-4582 (1985). 